\title{
Ranibizumab and Risk of Hospitalisation for Ischaemic Stroke and Myocardial Infarction in Patients with Age-Related Macular Degeneration: A Self-Controlled Case-Series Analysis
}

\author{
Nicole L. Pratt • Emmae N. Ramsay • Anna Kemp • \\ Lisa M. Kalisch-Ellett • Sepehr Shakib • Gillian E. Caughey • \\ Philip Ryan - Stephen Graves $\cdot$ Elizabeth E. Roughead
}

Published online: 27 September 2014

(C) The Author(s) 2014. This article is published with open access at Springerlink.com

\begin{abstract}
Background Ranibizumab, a vascular endothelial growth factor (VEGF) inhibitor, is used in the treatment of agerelated macular degeneration. Inhibition of VEGF has an anti-angiogenic action and is associated with thrombogenicity, thus, myocardial infarction and ischaemic stroke are potential side effects of VEGF inhibitors.

Objective Our objective was to assess the association between use of ranibizumab and risk of hospitalisation for ischaemic stroke (IS) and myocardial infarction (MI).

Methods The self-controlled case series design was used, including subjects exposed to ranibizumab (Anatomical
\end{abstract}

N. L. Pratt · E. N. Ramsay · L. M. Kalisch-Ellett ·

G. E. Caughey · E. E. Roughead

School of Pharmacy and Medical Sciences, University of South

Australia, Adelaide, SA, Australia

N. L. Pratt $(\square)$

Quality Use of Medicines and Pharmacy Research Centre,

University of South Australia, GPO Box 2471, Adelaide,

SA 5001, Australia

e-mail: Nicole.pratt@unisa.edu.au

\section{A. Kemp}

Centre for Health Services Research, The University of Western Australia, Crawley, Australia

\section{S. Shakib}

Department of Clinical Pharmacology, Royal Adelaide Hospital, Adelaide, SA, Australia

\section{P. Ryan}

Data Management and Analysis Centre, Discipline of Public

Health, University of Adelaide, Adelaide, SA, Australia

\section{S. Graves}

Australian Orthopaedic Association National Joint Replacement Registry, Adelaide, SA, Australia
Therapeutic Chemical [ATC] code S01LA04) who were hospitalized for IS (International Classification of Diseases, tenth edition [ICD-10] code I63) or the combined endpoint of stroke or transient ischaemic attack (TIA) (ICD-10 code G45) or MI (ICD-10 code I21) were identified between August 2007 and March 2013. Rate ratios in exposed periods compared with unexposed periods were calculated using conditional Poisson regression.

Results A total of 323 subjects received ranibizumab and were hospitalized for IS, 490 for IS or TIA, and 391 for MI. Median period of exposure was 8-9 months with follow-up times of approximately 2.8 years. No elevated risk of IS was seen in the 1-30 days post initiation (incidence rate ratio [IRR] 1.36; $95 \%$ confidence interval [CI] 0.98-1.88); however, elevated risk was observed for those who received therapy for 31-60 days (IRR 1.91; $95 \%$ CI 1.13-3.24). Sensitivity analyses adjusting for time-varying confounders found elevated risk in both the 1-30 days and

\section{Key Points}

A significantly increased risk of hospitalisation for ischaemic stroke was observed 31-60 days post ranibizumab injection.

No increased risk of hospitalisation for myocardial infarction was observed in any time period post ranibizumab injection.

Whilst the absolute risk of ischaemic stroke is likely to be small, clinicians should be aware of the potential for increased risk of stroke with ranibizumab. 
31-60 days periods. Similar results to those for IS were observed for the combined endpoint of IS or TIA. No association was seen for MI in either time period (1-30 days IRR $0.90,95 \%$ CI $0.65-1.23 ; 31-60$ days IRR $0.98,95 \%$ CI $0.54-1.79)$.

Conclusion This case-series analysis suggests an increased risk of hospitalisation for ischaemic stroke for patients receiving ranibizumab in the 31-60 days risk period. Studies with larger populations are required to confirm the risk in the 1-30 days risk period. No evidence of increased risk of hospitalisation for MI was observed.

\section{Introduction}

Ranibizumab, a vascular endothelial growth factor (VEGF) inhibitor, is used in the treatment of age-related macular degeneration [1]. VEGF stimulates the production of nitric oxide, which has multiple vasculoprotective effects, including vasodilation, antithrombotic activity and angiogenesis [2]. Inhibition of VEGF has an anti-angiogenic action and is associated with thrombogenicity, thus, myocardial infarction (MI) and ischaemic stroke (IS) are potential side effects of VEGF inhibitors [2]. The initial ranibizumab trials, on which market registration was granted, highlighted the potential safety issue of thromboembolic events. The ANCHOR clinical trial showed a higher rate of arterio-thromboembolic events in those receiving $0.5-\mathrm{mg}$ doses of ranibizumab compared with the $0.3-\mathrm{mg}$ dose or verteporfin injection $(4.3 \%$ in the $0.5-\mathrm{mg}$ group vs. 2.2 and $2.1 \%$ in the $0.3-\mathrm{mg}$ and verteporfin groups, respectively) [3]. The MARINA clinical trial showed rates of arterio-thromboembolic events were $4.6 \%$ in those treated with 0.3 or $0.5 \mathrm{mg}$ ranibizumab compared with $3.8 \%$ in those who received sham injections [4]. A 2009 meta-analysis of the data from the MARINA, ANCHOR and FOCUS trials pooled data for 859 subjects treated with ranibizumab (either 0.5 or $0.3 \mathrm{mg}$ ) and 454 treated with sham injections. The meta-analysis found an increased but non-significant risk of cerebrovascular accident (CVA) in those receiving monthly ranibizumab injections compared with sham injections (odds ratio [OR] 3.24 [95\% confidence interval (CI) $0.96-10.95]$ ). No association was observed for MI (OR 0.61 [95\% CI 0.29-1.29]) or the combined endpoint of MI or CVA (OR 1.08 [95 \% CI 0.59-1.98]) [5]. Another meta-analysis also showed no increased risk from trial data when assessing the combined endpoint of MI and stroke between ranibizumab and bevacizumab (relative risk [RR] 0.80 [95\% CI 0.30-2.13]) [6]. A 2012 pooled analysis using data from five trials showed no statistically significant increase in risk of cerebrovascular events when comparing the different doses against placebo (ranibizumab $0.5 \mathrm{mg}$ vs. control OR
2.2 [95 \% CI 0.8-7.1]; ranibizumab $0.3 \mathrm{mg}$ vs. control OR 1.2 [95\% CI 0.4-4.4]) or against each other (0.5 vs. $0.3 \mathrm{mg}$ OR 1.5 [95\% CI 0.8-3.0]) [7]. However, there was a sevenfold (OR 7.7 [95\% CI 1.2-177]) increased risk of stroke with $0.5 \mathrm{mg}$ ranibizumab compared with control in patients who were at high risk for CVA [7].

A large observational cohort study used administrative claims data to assess the adverse events of death, haemorrhage, MI and stroke associated with VEGF inhibitors. It involved almost 147,000 subjects and found a reduced risk of MI but no difference in stroke risk when ranibizumab was compared with photodynamic therapy, pegatinib or bevacizumab [8]. Stroke outcomes assessed included the combined endpoint of haemorrhagic stroke or IS. IS was not reported separately. A smaller Australian cohort study involving just under 3,500 patients found an increased risk of MI in those who attended an eye clinic and received VEGF inhibitors compared with randomly selected community controls, but no difference in those who attended the eye clinic and received photodynamic therapy. No difference in IS was observed [9]. While the study adjusted for comorbidities, the community cohort had greater prior stroke risk, and the extent of residual confounding was unknown. A nested case-control study compared exposure to VEGF inhibitors in the 180-day period before incident stroke or MI. The study included 1,477 stroke cases, 2,229 MI cases and event-free controls matched 5:1. No differences in exposure to VEGF inhibitors were observed between cases and controls [10]. An ecological study assessing trends in stroke rates among the population with diagnosed retinal disease reported no change in strokes after introduction of the VEGF inhibitors [11].

The assessment of thromboembolic events as adverse events of ranibizumab is complicated for two reasons: (1) stroke and MI are uncommon events, thus large sample sizes are required to detect differences, and (2) people with macular degeneration have comorbid conditions that place them at increased risk of stroke and MI, thus potentially confounding cohort or case-control studies. To overcome the potential for this confounding, we chose to assess the association using the self-controlled case-series method [12, 13]. The main advantage of using this design is that it minimises confounding because the patient acts as their own control. The within-person design controls implicitly for fixed known and unknown confounders, such as background cerebrovascular risk. Potential confounders that vary over time, such as age, can be adjusted for within the model. The design only includes individuals who have the outcome of interest and who were exposed to the medicine, comparing time on the medicines with time not exposed. While ranibizumab has a suggested monthly dosing schedule, therapy is often provided intermittently [14, 15], meaning subjects have both exposed and unexposed time to contribute. 
The aim of this study was to determine the association between ranibizumab and hospitalisation for IS and MI using the self-controlled case-series method.

\section{Methods}

The data source for this study was the Australian Department of Veterans' Affairs (DVA) administrative claims databases. DVA claims data contain records of prescription medicines dispensed under the Repatriation Pharmaceutical Benefits Scheme, medical and allied health services and hospital admissions provided to clients for whom DVA pays a subsidy. Across the time period analysed, approximately 300,000 clients were eligible for inclusion in the study. A client file is maintained by DVA that includes data on gender, date of birth, date of death and family status.

We looked at two primary outcomes: (1) hospitalisation for IS (International Classification of Diseases, tenth edition [ICD-10] code I63), and (2) combined endpoint of IS or transient ischaemic attack (TIA) (ICD-10 code G45) and (3) hospitalisation for MI (ICD-10 code I21). The exposure of interest for this study was a dispensing of ranibizumab (Anatomical Therapeutic Chemical [ATC] code S01LA04). Only those who had been dispensed ranibizumab during the study period were included in the study cohort, as macular degeneration itself is associated with an increased risk of stroke. To ensure we had 'new users', the study period was the first date that a ranibizumab prescription appeared in the database, 1 August 2007, and the study end was 31 March 2013. To be eligible for the study, subjects needed to be aged between 45 and 100 years of age at 1 August 2007, have at least one prescription medicine in the 12 months prior to entry into the cohort and be eligible for all health services subsidised by DVA.

All ranibizumab scripts dispensed during the study period were obtained for included individuals. Since dosage information is not available in the dataset, a patient was considered 'exposed' to ranibizumab for a period of 30 days after the supply date. This assumption is consistent with its clinical use and duration of therapeutic effect. A 30-day duration of exposure was applied to each dispensing of ranibizumab. Patients with repeat dispensings within 30 days were considered continuously exposed. If there was no supply of ranibizumab within the 30 days, the patient was considered no longer exposed from 30 days after the last date of supply. The actual date of the injection was unknown, and this analysis assumes the injection was the day of supply. Person time was then divided into preand post-exposure risk periods. The time after initiation of ranibizumab was divided into the following risk periods: day of supply, 1-30 days, 31-60 days, $>60$ days and a wash-out period of 1-30 days starting the day after a subject was considered to be no longer exposed. Patients would contribute person time to the 31-60 days risk period only if they had two dispensings of ranibizumab within 30 days of each other. These risk periods were chosen a priori to be consistent with dosage recommendation for ranibizumab, which is one injection per month. Additionally, two consecutive 42-day pre-exposure risk periods were included prior to the very first supply of ranibizumab only during the study period. These time periods were based on rehabilitation times for stroke recovery, which is a time period in which ranibizumab is unlikely to be used. All remaining time was considered unexposed to ranibizumab.

In each risk period, the hospitalisation rate, the cumulative number of hospitalisations divided by the personyears at risk in that period, was compared with the rate of hospitalisations in the unexposed risk period. If a subject was re-hospitalised within 30 days, the subsequent hospitalisation(s) were excluded, as they were considered to be part of the same episode [12]. Incident rate ratios (IRRs) were calculated using conditional Poisson regression, with results presented as adjusted rate ratios and $95 \%$ CIs. Since the self-controlled case-series method controls implicitly for fixed covariates $[12,13]$, the primary analyses were adjusted for time-varying age only.

We also performed sensitivity analyses where we (1) adjusted for potential time-varying confounders, and (2) included just those patients who survived at least 90 days after their hospitalisation event. The following time-varying confounders were considered: number of prescriptions, number of hospitalisations and number of comorbid conditions (using the validated Rx-Risk-V score [16]), which were determined annually. A time-varying indicator variable was used for exposure to the following medicines: a non-steroidal anti-inflammatory drug (NSAID) (M01A), an antithrombotic (B01A) and an antihypertensive (C09, C08, C07, C10BX, C03, C02), and adjustment was made for cancer (hospitalisation ICD code C or D00-D48 or a dispensing of L01 medicine) and renal failure (ICD code N17N19) where the indicative hospitalisation or medicine use occurred in the previous 12 months. Ethics approval was obtained from the University of South Australia Human Research Ethics Committee and the DVA Human Research Ethics Committee. All analyses were performed using SAS version 9.4 (SAS Institute, Cary, NC, USA).

\section{Results}

During the study period, 323 subjects received ranibizumab and experienced an IS, 490 experienced an IS or TIA, and 391 an MI. Characteristics of the study cohort are presented in Table 1. 
Table 1 Characteristics of study subjects

\begin{tabular}{lcll}
\hline & IS & IS or TIA & MI \\
\hline Number of subjects & 323 & 490 & 391 \\
Follow-up years & 2.7 & 2.8 & 2.9 \\
& $(1.4-4.0)$ & $(1.5-4.1)$ & $(1.5-4.2)$ \\
Duration of exposure (years) & 0.7 & 0.7 & 0.7 \\
& $(0.3-1.3)$ & $(0.3-1.5)$ & $(0.3-1.5)$ \\
Age at first exposure (years) & $85(82-87)$ & $84(82-87)$ & $84(82-86)$ \\
Age at first hospitalisation & $87(85-90)$ & $87(85-90)$ & $87(84-90)$ \\
$\quad$ years) & & & \\
Male $(\%)$ & 47 & 44 & 55 \\
\hline
\end{tabular}

Data are presented as median (range) unless otherwise indicated

$I S$ ischaemic stroke, MI myocardial infarction, TIA transient ischaemic attack

The risk of IS was elevated but not statistically significant in the first 30 days after supply (IRR 1.36 [95\% CI 0.98-1.88]); however, the risk was significantly increased in the 31-60 days after first supply (IRR 1.91 [95\% CI 1.13-3.24]) (Table 2). The risk of stroke for those exposed for more than 60 days was similar to that for unexposed patients (IRR 0.73 [95\% CI 0.26-2.07]). For the combined outcome of IS or TIA, similar results were observed to the analysis for IS only. No increased risk of MI was observed in any of the risk periods (Table 2).

The sensitivity analyses, results for the risk of IS were similar after adjusting for time-varying confounders; however, the risk was statistically significant in the 1-30 days risk period (IRR 1.38 [95\% CI 1.00-1.91]) and the 31-60 days risk period (IRR 1.97 [95\% CI 1.16-3.35]). When only those patients who had survived for at least 90 days after their admission to hospital for the event of interest were analysed, results were similar, with a significantly increased risk in the 31-60 days risk period (IRR 2.10 [95\% CI 1.20-3.69]). No significant association was found in any of the sensitivity analyses for the outcome of MI.

\section{Discussion}

The results of this self-controlled case-series study suggest an elevated risk of IS in patients who remain on ranibizumab therapy between 31 and 60 days post-dispensing. Adjustment for time-varying confounders found stroke risk elevated at both 1-30 days and 31-60 days. We found no evidence of increased risk of MI. No significant increased risk for IS was detected beyond 60 days of continuous therapy. Our results differ from those of previous observational studies, which did not find an increased risk of stroke [8, 9]; however, our results are consistent with randomised controlled trial evidence, which found a statistically non-significant increased risk of stroke and no increased risk of MI [5].

Observational studies, such as the cohort study design, have the potential for residual confounding, which is a particular issue for the population with macular degeneration, as common conditions comorbid to macular degeneration increase the risk of MI and stroke independently of any potential effect of the medicine under study. One of the advantages of the self-controlled case series design is that it controls implicitly for patient-specific confounders that do not vary over time. This means that it is not necessary to adjust for variables such as gender, frailty or other risk factors for stroke that are constant over time. Our study only included people who had received ranibizumab and who had a stroke, thus limiting confounding due to differences in comorbid conditions. Further, our sensitivity analysis, where we adjusted for comorbidities in a timevarying manner, did not change the results substantially, suggesting the design itself adequately controlled for confounding. The assessment of the risk of stroke with ranibizumab also meets the assumptions of the method, which are that exposure is intermittent, the outcome is acute and the occurrence of the outcome, stroke, is unlikely to influence the exposure, ranibizumab. This means that hospital events must not increase the probability of death. We were unable to determine causes of death from our data, so we could not estimate how many patients died as a result of stroke. Farrington and Whitaker [17] have shown that the case-series method may be robust to failure of this assumption and may result in small negative bias. In this example, only $11 \%$ of the hospitalisations for stroke resulted in death at discharge, which suggests that the results should be robust to the assumption. As a sensitivity analysis, we included only those patients who had survived at least 90 days after their stroke, and risk estimates were similar in this group to those of the primary analysis.

Only one formulation of ranibizumab is subsidised in Australia, and dosage information is not recorded in the dataset, thus we were unable to do an analysis by dose. The initial trials reported a higher risk of stroke with higher dose of ranibizumab [3]. Another limitation of our study was that we could not determine whether patients received injections in one eye only or received bilateral injections. Bilateral treatment has been reported for between 5 and $20 \%$ of patients [18]. The claims data enabled assessment of a record of an intravitreal injection, but not the medicine administered by the injection. Intravitreal injection (Medicare Benefits Schedule [MBS] code 42740) is not unique for ranibizumab. Very few of the dispensings of ranibizumab included in this study could be matched within a 2-day window to the MBS service claim for intravitreal injection. Since we were unable to identify the actual injection date, our assumed day of exposure, i.e. 
Table 2 Risk of ischaemic stroke, myocardial infarct, or ischaemic stroke or transient ischaemic attack before and after ranibizumab supply

\begin{tabular}{|c|c|c|c|c|c|c|}
\hline Risk periods & $N$ & Person-years & Rate per 10 years & $\operatorname{IRR}^{\mathrm{a}}$ & $\mathrm{IRR}^{\mathrm{b}}$ & $\operatorname{IRR}^{\mathrm{c}}$ \\
\hline \multicolumn{7}{|c|}{ Hospitalisation for ischaemic stroke } \\
\hline Unexposed & 194 & 1023 & $1.94(1.67-2.26)$ & 1.00 & 1.00 & 1.00 \\
\hline Pre $43-84$ days & 3 & 31 & $0.95(0.30-2.94)$ & $0.49(0.16-1.53)$ & $0.51(0.16-1.60)$ & $0.61(0.19-1.91)$ \\
\hline Pre $1-42$ days & 5 & 35 & $1.43(0.59-3.45)$ & $0.74(0.30-1.80)$ & $0.76(0.31-1.87)$ & $0.92(0.38-2.25)$ \\
\hline Same day & 2 & 8 & $2.37(0.59-9.51)$ & $1.22(0.30-4.95)$ & $1.25(0.31-5.08)$ & $0.77(0.11-5.54)$ \\
\hline Post $1-30$ days & 65 & 239 & $2.64(2.02-3.46)$ & $1.36(0.98-1.88)$ & $1.38^{\dagger}(1.00-1.91)$ & $1.32(0.92-1.90)$ \\
\hline Post $31-60$ days & 17 & 45 & $3.71(2.26-6.10)$ & $1.91^{\dagger}(1.13-3.24)$ & $1.97^{\dagger}(1.16-3.35)$ & $2.10^{\dagger}(1.20-3.69)$ \\
\hline Post $>60$ days & 4 & 28 & $1.43(0.52-3.95)$ & $0.73(0.26-2.07)$ & $0.81(0.29-2.29)$ & $0.66(0.20-2.15)$ \\
\hline Wash-out period & 33 & 164 & $1.90(1.32-2.72)$ & $0.98(0.65-1.46)$ & $0.99(0.66-1.49)$ & $1.04(0.67-1.62)$ \\
\hline \multicolumn{7}{|c|}{ Hospitalisation for ischaemic stroke or transient ischaemic attack } \\
\hline Unexposed & 291 & 1584 & $1.90(1.68-2.15)$ & 1.00 & 1.00 & 1.00 \\
\hline Pre 43-84 days & 5 & 48 & $1.05(0.43-2.52)$ & $0.55(0.23-1.34)$ & $0.57(0.23-1.38)$ & $0.64(0.26-1.55)$ \\
\hline Pre 1-42 days & 11 & 53 & $2.09(1.16-3.79)$ & $1.10(0.60-2.02)$ & $1.12(0.61-2.05)$ & $1.28(0.70-2.36)$ \\
\hline Same day & 4 & 13 & $3.04(1.14-8.14)$ & $1.60(0.59-4.33)$ & $1.66(0.61-4.48)$ & $1.41(0.45-4.43)$ \\
\hline Post $1-30$ days & 94 & 387 & $2.46(1.97-3.06)$ & $1.29(0.99-1.69)$ & $1.34^{\dagger}(1.02-1.74)$ & $1.29(0.97-1.72)$ \\
\hline Post $31-60$ days & 27 & 76 & $3.64(2.46-5.40)$ & $1.92^{\dagger}(1.26-2.92)$ & $1.96^{\dagger}(1.29-2.99)$ & $2.05^{\dagger}(1.33-3.18)$ \\
\hline Post $>60$ days & 5 & 43 & $1.17(0.48-2.88)$ & $0.62(0.25-1.54)$ & $0.66(0.27-1.65)$ & $0.57(0.20-1.56)$ \\
\hline Wash-out period & 53 & 264 & $1.98(1.49-2.63)$ & $1.04(0.76-1.44)$ & $1.07(0.78-1.48)$ & $1.11(0.79-1.56)$ \\
\hline \multicolumn{7}{|c|}{ Hospitalisation for myocardial infarction } \\
\hline Unexposed & 254 & 1275 & $2.17(1.90-2.47)$ & 1.00 & 1.00 & 1.00 \\
\hline Pre $43-84$ days & 7 & 39 & $1.78(0.84-3.75)$ & $0.82(0.39-1.75)$ & $0.87(0.41-1.85)$ & $0.99(0.46-2.10)$ \\
\hline Pre $1-42$ days & 5 & 43 & $1.13(0.47-2.72)$ & $0.52(0.21-1.27)$ & $0.54(0.22-1.31)$ & $0.64(0.26-1.55)$ \\
\hline Same day & 5 & 10 & $4.87(2.02-11.77)$ & $2.25(0.92-5.51)$ & $2.22(0.91-5.46)$ & $2.21(0.81-6.01)$ \\
\hline Post $1-30$ days & 58 & 298 & $1.94(1.48-2.56)$ & $0.90(0.65-1.23)$ & $0.88(0.64-1.21)$ & $0.97(0.69-1.37)$ \\
\hline Post $31-60$ days & 12 & 57 & $2.13(1.19-3.80)$ & $0.98(0.54-1.79)$ & $1.01(0.55-1.85)$ & $0.91(0.46-1.82)$ \\
\hline Post $>60$ days & 4 & 30 & $1.28(0.46-3.54)$ & $0.59(0.21-1.65)$ & $0.61(0.22-1.70)$ & $0.75(0.27-2.12)$ \\
\hline Wash-out period & 46 & 201 & $2.28(1.68-3.09)$ & $1.05(0.74-1.48)$ & $1.01(0.71-1.43)$ & $0.94(0.64-1.40)$ \\
\hline
\end{tabular}

Figures in parentheses are $95 \%$ confidence intervals

ICD International Classification of Diseases, IRR incidence rate ratio

+ Statistically significant

a Adjusted for age

b Adjusted for age, number of prescriptions, number of hospitalisations and number of comorbid conditions (using the validated Rx-Risk-V score [16]), which were determined annually. A time-varying indicator variable was used for exposure to the following medicines: a nonsteroidal anti-inflammatory drug (M01A), an antithrombotic (B01A) and an antihypertensive (C09, C08, C07, C10BX, C03, C02), and adjustment was made for cancer (hospitalisation ICD code C or D00-D48 or a dispensing of L01 medicine) and renal failure (ICD code N17$\mathrm{N} 19)$, where the indicative hospitalisation or medicine use occurred in the previous 12 months

c Analysis in only those patients who survived at least 90 days after their hospitalisation event

dispensing date, may be before the medicine was actually administered. This may partly explain the lower risk of stroke in the 0-30 days risk period if, in fact, some of the patients had a delay between dispensing and administration. We included only those patients who had at least one dispensing of ranibizumab during the study period. During the study period, ranibizumab was only subsidised in Australia for treatment of aged-related macular degeneration as diagnosed by fluorescein angiography. More than half of the population included in this study had initiated ranizibumab within 2 years of study entry and are likely to have pre-existing macular degeneration during this time, particularly as the treatment was first subsidised in 2007 (study entry date). For some patients, it is possible that unexposed person-time may include time in which they did not have macular degeneration and therefore may be at lower risk of stroke. Including this time may have biased results away from the null; however, since the study period is short, this is unlikely to be an issue in this study. Veterans in Australia receive their health services through Australia's universal health system. Australia does not have separate health services for veterans, thus our results are likely to reflect Australian clinical practice, not veteranspecific care. Our study was undertaken in the Australian 
veteran population, which is an elderly population and, whilst the results are likely to be generalizable to other elderly populations, the applicability of our results to younger individuals is unknown. Analysis of data on 4,444 patients enrolled in European registries of macular degeneration reported an average age of patients of 78-79 years [18], which is slightly younger than our population. While the risk of IS was significantly increased in the 31-60 days risk period, the number of stroke events was only 17 . This suggests that the absolute risk of IS is low. In the primary analysis, we included only those patients who had an IS hospitalisation and were dispensed ranibizumab. A total of 7,898 patients had at least one ranibizumab dispensing during the study period, equating to rates in the population with macular degeneration of 4 per 100 for IS, 6.2 per 100 for IS or TIA and 5.0 for MI. This is consistent with studies showing elevated stroke risk in those with macular degeneration of 1.5 times that of the general population [19], which equates to approximately $2.5 \%$ in the general population over 85 years [20].

\section{Conclusion}

This self-controlled case-series analysis suggests an increased risk of IS with ranibizumab in the 31-60 days risk period. Studies with larger populations are necessary to confirm the risk in the 1-30 days period. We found no evidence of increased risk of MI. Our results suggest that prescribers should be vigilant for stroke risk in their patients.

Financial support This work was supported by an Australian Government National Health and Medical Research Council Centre of Research Excellence in Post-Marketing Surveillance of Medicines and Medical Devices grant (Grant Number APP1040938). NP is supported by an NHMRC Early Career Fellowship (Grant Number GNT1035889).

Conflict of interest Nicole L. Pratt, Emmae N. Ramsay, Anna Kemp, Lisa M. Kalisch-Ellett, Sepehr Shakib, Gillian E. Caughey, Philip Ryan, Stephen Graves and Elizabeth E. Roughead have no conflicts of interest that are directly relevant to the content of this study.

Open Access This article is distributed under the terms of the Creative Commons Attribution Noncommercial License which permits any noncommercial use, distribution, and reproduction in any medium, provided the original author(s) and the source are credited.

\section{References}

1. Australian Medicines Handbook Pty Ltd. Australian medicines handbook. In: Rossi S, editor. Adelaide: Australian Medicines Handbook Pty Ltd; 2003.
2. Tunon J, Ruiz-Moreno JM, Martin-Ventura JL, Blanco-Colio LM, Lorenzo O, Egido J. Cardiovascular risk and antiangiogenic therapy for age-related macular degeneration. Surv Ophthalmol. 2009;54(3):339-48.

3. Brown DM, Kaiser PK, Michels M, Soubrane G, Heier JS, Kim $\mathrm{RY}$, et al. Ranibizumab versus verteporfin for neovascular agerelated macular degeneration. N Eng J Med. 2006;355(14): 1432-44.

4. Chang TS, Bressler NM, Fine JT, Dolan CM, Ward J, Klesert TR, et al. Improved vision-related function after ranibizumab treatment of neovascular age-related macular degeneration: results of a randomized clinical trial. Arch Ophthalmol. 2007;125(11): 1460-9.

5. Ueta T, Yanagi Y, Tamaki Y, Yamaguchi T. Cerebrovascular accidents in ranibizumab. Ophthalmology. 2009;116(2):362.

6. Schmucker C, Ehlken C, Agostini HT, Antes G, Ruecker G, Lelgemann $\mathrm{M}$, et al. A safety review and meta-analyses of bevacizumab and ranibizumab: off-label versus goldstandard. PLoS One. 2012;7(8):e42701.

7. Bressler NM, Boyer DS, Williams DF, Butler S, Francom SF, Brown B, et al. Cerebrovascular accidents in patients treated for choroidal neovascularization with ranibizumab in randomized controlled trials. Retina. 2012;32(9):1821-8.

8. Curtis LH, Hammill BG, Schulman KA, Cousins SW. Risks of mortality, myocardial infarction, bleeding, and stroke associated with therapies for age-related macular degeneration. Arch Ophthalmol. 2010;128(10):1273-9.

9. Kemp A, Preen DB, Morlet N, Clark A, McAllister IL, Briffa T, et al. Myocardial infarction after intravitreal vascular endothelial growth factor inhibitors: a whole population study. Retina. 2013;33(5):920-7.

10. Campbell RJ, Gill SS, Bronskill SE, Paterson JM, Whitehead M, Bell CM. Adverse events with intravitreal injection of vascular endothelial growth factor inhibitors: nested case-control study. BMJ. 2012;345:e4203.

11. Campbell RJ, Bell CM, Paterson JM, Bronskill SE, Moineddin R, Whitehead M, et al. Stroke rates after introduction of vascular endothelial growth factor inhibitors for macular degeneration: a time series analysis. Ophthalmology. 2012;119(8):1604-8.

12. Whitaker HJ, Farrington CP, Spiessens B, Musonda P. Tutorial in biostatistics: the self-controlled case series method. Stat Med. 2006;25(10): 1768-1797.

13. Whitaker HJ, Hocine MC, Farrington CP. The methodology of self-controlled case series studies. Stat Methods Med Res. 2009;18:7-26.

14. Cohen SY, Dubois L, Tadayoni R, Fajnkuchen F, Nghiem-Buffet S, Delahaye-Mazza C, et al. Results of one-year's treatment with ranibizumab for exudative age-related macular degeneration in a clinical setting. Am J Ophthalmol. 2009;148(3):409-13.

15. Geirsdottir A, Jonsson O, Thorisdottir S, Helgadottir G, Jonasson F, Stefansson E, et al. Population-based incidence of exudative age-related macular degeneration and ranibizumab treatment load. Br J Ophthalmol. 2012;96(3):444-7.

16. Sloan K, Sales A, Liu C, Fishman P, Nichol P, Suzuki N, et al. Construction and characteristics of the RxRisk-V: a VA-adapted pharmacy-based case-mix instrument. Med Care. 2003;41(6): 761-74.

17. Farrington $P$, Whitaker H. Mortality and the self-controlled case series method: letter to the editor. Pharmacoepidemiol Drug Saf. 2012;21(8):906 (author reply 7).

18. Holz FG, Bandello F, Gillies M, Mitchell P, Osborne A, Sheidow $\mathrm{T}$, et al. Safety of ranibizumab in routine clinical practice: 1-year retrospective pooled analysis of four European neovascular AMD registries within the LUMINOUS programme. Br J Ophthalmol. 2013;97(9):1161-7. 
19. Islam MS, Anderson CS, Hankley GJ, Hardie K, Carter K, Broadhurst R, Jamrozik K. Trends in incidence and outcome of stroke in Perth, Western Australia During 1989 to 2001: The Perth Community Stroke Study. Stroke. 2008;39:776-82.
20. Ikram MK, Mitchell P, Klein R, Sharrett R, Couper DJ, Wong TY. Age-related macular degeneration and long-term risk of stroke subtypes. Stroke. 2012;43:1681-3. 